\title{
Hierarchie oder Netzwerk? Zu den Organisationsformen des Terrorismus
}

Auf der Grundlage einer ausgedehnten Literatursuche werden in diesem Aufsatz die Organisationsformen des Terrorismus in Kategorien der Organisationssoziologie analysiert. In der Literatur wird der hierarchisch organisierte, auf ein nationales Territorium bezogene „alte" Terrorismus von Organisationen wie ETA, IRA oder Hamas den neuen, vor allem mit dem Namen Al Qaida verbundenen „Netzwerken des Terrors" gegenübergestellt. Tatsăchlich weisen jedoch alle verdeckt operierenden Terrororganisationen eine Kombination von hierarchischen und Netzwerkmerkmalen auf. Darüber hinaus weist der organisierte Terrorismus eine Reihe von Merkmalen auf, die weder ins hierarchische Muster passen noch allgemein netzwerktypisch sind. Dabei handelt es sich um einen besonderen Steuerungsmodus und einen besonderen Modus loser Koppelung, die beide auf einer starken ideologischen Identifikation der Mitglieder mit der Organisation und ihren Zielen beruhen. Die Identifikation dieser besonderen Merkmale terroristischer Organisation leistet einen Beitrag zur Theorie der Handlungskoordination, speziell in interorganisatorischen Netzwerken.

\section{Fragestellung und Datenbasis}

Überraschende historische Ereignisse wie die Auflösung der UdSSR, die deutsche Vereinigung oder die Anschläge vom 11. September 2001 stellen regelmaßig Herausforderungen für die Sozialwissenschaftler dar und fordern sie dazu auf, die Pfade der normal science zu verlassen und ein neues Thema aufzugreifen. Wenn sie das tatsăchlich tun, benutzen sie zunächst ihr vertrautes Begriffswerkzeug, das sie dann in der Auseinandersetzung mit den Ursachen, Erscheinungsformen oder Folgen des neuen Phänomens verwerfen, modifizieren oder erweitern. So ist das kurz als ,9/11" bezeichnete Ereignis der Anschläge von New York und Washington von der deutschen Politikwissenschaft vor allem im Rahmen der Diskussion über den Wandel des Krieges und neue Formen gewalttätiger Austragung von Konflikten (vgl. Münkler 1992; Waldmann 1993; Münkler 2001), gelegentlich auch im Zusammenhang mit traditionellen Ordnungskonzeptionen von Politik (Behr 2002) behandelt worden. In der Soziologie müsste sich die Bewegungsforschung herausgefordert sehen, die UUbergänge von einer sozialen Protestbewegung entweder zu legalen politischen oder zu im Untergrund arbeitenden terroristischen Organisationen intensiv zu dis- kutieren. Organisationssoziologie und Netzwerkforschung dagegen sollten sich mit der in Analysen des "neuen Terrorismus" vie]fach geäußerten Behauptung eines Wechsels von hierarchischen zu netzwerkartigen Organisationsformen des Terrorismus auseinandersetzen. Genau dies ist denn auch die Absicht dieses Aufsatzes. Aufgrund meiner Beschäftigung mit Organisationssoziologie (Mayntz 1963) und meines Interesses an Interorganisationsnetzwerken (Marin/Mayntz 1991; Mayntz 1993) bewog mich das Ereignis des 9. September 2001 spontan dazu, ein für mich völlig neues Thema aufzugreifen und in der Literatur über "alten" und „neuen“ Terrorismus nach Aussagen über seine verschiedenen Organisationsformen $\mathrm{zu}$ suchen. Zugegeben, dieses Erkenntnisinteresse ist ein sehr spezielles, ja vielleicht herzlos „akademisch" anmutendes, spart es doch die aus schockierter Betroffenheit heraus meist gestellten Fragen nach dem Warum der jüngsten Welle von Terroranschlägen und nach der - jenseits des Erfahrungshorizonts des zufrieden in der legalen Welt lebenden Bürgers liegenden und kaum nachvollziehbaren - Geisteshaltung von Terroristen allgemein und von Selbstmordattentätern im Besonderen vollständig aus. Diesen naheliegenden Fragen widmet sich ein großer Teil vor allem der Literatur zum neuen internationalen Terrorismus (vgl. hierzu lediglich Reich 
1990, Elwert 2002). Hier soll dagegen gezeigt werden, wie die mit dem Phänomen Terrorismus bislang überhaupt nicht befasste Organisationssoziologie damit umgehen kann. In welchen Formen tritt organisierter Terrorismus auf? Lassen sich seine Organisationsformen in vertrauten analytischen Kategorien erfassen oder haben wir es mit einem Organisationsphänomen sui generis zu tun?

Grundlage meiner Literatursuche war in erster Linie eine 200 Titel umfassende annotierte Bibliographie zum internationalen Terrorismus, die am Lehrstuhl von Herfried Münkler auf der Grundlage eines Werkvertrags des Kölner Max-Planck-Instituts für Gesellschaftsforschung erarbeitet und im Dezember 2002 abgeschlossen wurde. Insgesamt habe ich rund 60 Bücher, Buchkapitel, Aufsätze und Berichte selber ausgewertet, 35 davon in Verfolg von Hinweisen in den Abstracts der genannten Bibliographie. Aus Platzgrunden wird diese Literatur hier nur äußerst sparsam zitiert. Die ausgewerteten Texte beziehen sich teils generell auf den islamistischen bzw. den "neuen" Terrorismus, überwiegend jedoch auf einzelne Organisationen, und zwar speziell auf die spanische ETA, die IRA, den peruanischen Leuchtenden Pfad (SL), die Tamil Tigers (LTTE), die PLO, Hamas, Hizbollah und Al Qaida.

Terrorismus ${ }^{1}$ kann sich in einer Vielzahl von Formen äußern. Neidhardt (1982) hat zwischen Einzeltätern, kleinen Netzen, hochintegrierten Gruppen und Organisationen unterschieden. Diese Typologie muss heute um die großen internationalen Netzwerke erweitert werden, die nicht einzelne Individuen, sondern Gruppen und Organisationen miteinander verbinden. Von diesem Spektrum an Formen sollen hier lediglich die beiden letzten, Organisationen und internationale Netzwerke, betrachtet werden. ${ }^{2}$ Die oft räumlich konzentrierten und relativ kleinen Gruppen bzw. interpersonellen Netzwerke, die für die ehemalige RAF (Neidhardt 1982; Straßner 2003), die Brigate Rosse (della Porta 1995) oder die rechtsradikalen amerikanischen Milizen (Abanes 1996) kennzeichnend sind, bleiben dagegen unberücksichtigt. Dieser Fokus ist hoch selektiv. Dekmejian (1995)
z.B. hat allein im Nahen und Mittleren Osten 176 verschiedene fundamentalistische islamische Gruppen erfasst; viele von ihnen sind sehr klein, und nur einige von ihnen sind genauer bezüglich ihrer Struktur untersucht worden und können als Organisationen im soziologischen Sinn dieses Begriffs bezeichnet werden.

Für den in diesem Zusammenhang vielfach zitierten Laqueur (1999) unterscheidet sich der moderne oder neue Terrorismus von den früher dominanten Formen vor allem durch seine religiőse (statt säkulare) Basis und Zielrichtung, seine internationale (statt nationale) Ausdehnung, seine netzwerkartige (statt hierarchische) Struktur und seine Bereitschaft zur Benutzung von Massenvernichtungswaffen. Im Folgenden werden Reichweite (Abschnitt 2) und Struktur des organisierten Terrorismus (Abschnitt 3) im Mittelpunkt stehen. Im weiteren Sinne gehören zum Thema Organisationsformen des Terrorismus auch die Fragen nach Rekrutierung und Finanzierung, die hier jedoch nicht behandelt werden.

\section{Nationaler und internationaler Terrorismus}

In der gegenwärtigen Diskussion wird oft stillschweigend ein kausaler Zusammenhang zwischen der islamischen Fundierung und der Internationalisierung des Terrorismus unterstellt. Tatsächlich besteht jedoch kein offensichtlicher Zusammenhang zwischen der (nationalen bzw. internationalen) Reichweite und der (säkularen bzw. religiösen) Basis von Terrorismus. Zur Zeit existiert zwar keine internationale Organisation mit säkularer sozialrevolutionärer Zielsetzung, wie es die kommunistische Internationale vielleicht einmal war, aber es gibt eine Reihe bekannter Terrororganisationen mit säkularem national-revolutionären Fokus, sowohl in nicht-islamischen (z.B. ETA, SL, LTTE) wie in islamischen Ländern (PFLP, PLO). Auch unter den religiös fundierten Gruppierungen gibt es solche mit nationalem und mit transnationalem Bezug. $\mathrm{Zu}$ ersteren gehört die fundamentalistische, einen islamischen Staat 
Palästina anstrebende Hamas oder auch der ägyptische Dschihad. Al Qaida dagegen hat keinen definierten nationalen Bezugspunkt mehr; verändert werden soll der status quo der islamischen (nicht nur arabischen!) Welt durch Bekämpfung des „Westens“ und speziell der USA, die die eigentlich Verantwortlichen für die jetzigen, als Missstand gedeuteten Verhältnisse in diesen Ländern sind (für eine politikwissenschaftliche Ursachenanalyse vgl. Kitschelt 2003). Die internationale Verbreitung des Islam, verbunden mit einer (wie auch immer zu erklärenden) Intensivierung von islamischer Religiosität, ist dabei sicher eine günstige Voraussetzung für eine internationale Ausbreitung des islamischen Terrorismus. Zugleich erlaubt ein umfassender und damit auch unscharfer Feindbegriff es, Unterschiede in der Zielsetzung verdeckt zu halten - eine organisationssoziologisch oft festgestellte Funktion unscharfer und damit verschieden interpretierbarer Zieldefinitionen. Sehr allgemein formulierte Ziele und ein unscharfer Feindbegriff begünstigen auch die Mobilisierung solcher Personen für terroristische Aktivităten, die unter dem Mantel ideologischer Rechtfertigung lediglich ein Ventil für ihre diffuse Aggressivität und mörderischen Impulse suchen.

Es gibt verschiedene Formen einer Erweiterung organisierten Terrors über den nationalen Rahmen hinaus, die in der Literatur zwar beschrieben, aber nicht systematisch unterschieden werden. Sinnvoll ist zunächst eine Unterscheidung zwischen internationalen und transnationalen Organisationsformen, wie sie Schneckener (2002) vornimmt. Beide Kategorien lassen sich jedoch weiter unterteilen. So gibt es drei unterschiedliche Formen der internationalen Ausweitung von Aktivitäten nationaler Terrororganisationen. An erster Stelle zu nennen ist die Ausweitung terroristischer Aktivitäten auf Ziele in bzw. Zielpersonen aus anderen Staaten. Der Zweck ist hier die Verfolgung des auf den eigenen Staat bezogenen politischen Ziels, furr das die Attentate international Aufmerksamkeit schaffen wollen. Diese Art der Internationalisierung kann mit dem Aufbau von Stützpunkten für derartige Aktivitäten in anderen Ländern gekoppelt sein. Ein gutes
Beispiel bieten die bei Schröm (2002) geschilderten Aktivităten der palästinensischen PFLP. Eine zweite Form der internationalen Ausweitung wird am besten durch das höchst erfolgreiche, auf der tamilischen Diaspora aufbauende internationale Unterstutzungsnetzwerk der LTTE illustriert, einer Organisation mit nationaler politischer Zielsetzung (vgl. z.B. Gunaratna 1998). Die Unterstutzung ist in legalen Formen organisiert; meist geht es um Finanzierung (Spendensammeln etc.) und die Beeinflussung der offentlichen Meinung im Ausland. Eine dritte Form der internationalen Ausweitung ist schließlich die aktive Unterstützung des Aufbaus von Terrorgruppen bzw. -organisationen mit eigener nationaler Zielsetzung in anderen Ländern; bekanntes Beispiel ist die gezielte Unterstiutzung des Irans beim Aufbau der Hizbollah im Libanon nach der gelungenen islamistischen Revolution im eigenen Land (vgl. Wege 1994; Ranstorp 1994).

Von diesen verschiedenen Formen der Internationalisierung eines auf nationale Ziele gerichteten Terrorismus zu unterscheiden sind verschiedene Formen transnationaler Organisation. An erster Stelle zu nennen ist hier die pragmatisch motivierte, fallweise Kooperation distinkter terroristischer Organisationen, die sich gegenseitig helfen, obwohl sie verschiedene Ziele haben. Für die fallweise Kooperation liefert u.a. wieder Schröm (2002) gute Beispiele. Solche Kooperationen lassen sich natürlich als (Interaktions-)Netze darstellen, doch handelt es sich dabei typischerweise nicht um dauerhafte, uber den einzelnen Anlass hinausgehende Beziehungen. Von der fallweisen Kooperation sind zweitens die auf Dauer angelegten internationalen Netzwerke zu unterscheiden, in denen verschiedene lokale bzw. nationale terroristische Gruppen in der Verfolgung eines übergeordneten gemeinsamen Ziels zusammenarbeiten. Theoretisch gibt es schließlich drittens die Möglichkeit einer transnationalen terroristischen Organisation, deren Ziel nicht mehr auf ein bestimmtes nationales Territorium bezogen ist. Eine solche Organisation könnte durch die Gründung von „Filialen" oder „Chapters" von einem Zentrum aus oder durch die Absorption existenter nationaler oder auch lokaler Gruppen ent- 
stehen, wobei die Initiative dazu sowohl von der Mutterorganisation wie von den „Beitrittskandidaten" ausgehen kann - Möglichkeiten, für die sich in der Literatur über internationale Nichtregierungsorganisationen Beispiele finden ließen.

A] Qaida wird vielfach als zentrales Beispiel, ja geradezu als ,paradigmatisch" fur den neuen transnationalen Terrorismus angesehen (Schneckener 2002: 19), dem in der Literatur gewöhnlich ein Netzwerkcharakter zugeschrieben wird. Dabei wird jedoch nicht systematisch zwischen einer "Organisation mit Netzwerkmerkmalen" und einem ,(interorganisatorischen) Netzwerk" unterschieden. ${ }^{3} \mathrm{Im}$ ersten Fall handeln die Untereinheiten einer Organisation relativ selbstăndig, besitzen jedoch eindeutig den Status von Mitgliedern und bleiben grundsätzlich zentralen Vorgaben unterworfen; eine solche Organisationsform findet man oft in multinationalen Unternehmen (vgl. Bartlett/ Goshal 1989). Im zweiten Fall kooperieren selbstständige soziale Einheiten, die sich nicht als Bestandteile der gleichen Organisation begreifen, auf freiwilliger Basis; das gilt zum Beispiel für Allianzen, Kartelle oder transnationale Produktionsnetzwerke aus selbständigen Firmen (vgl. u.a. die Beiträge in Hollingsworth/Schmitter/Streeck 1994). In der Realität des organisierten Terrors mag die Grenze zwischen beiden Formen allerdings fließender sein als im Bereich rechtlich verfasster Unternehmen. Was Al Qaida angeht, fehlt jedenfalls die Information, um zu entscheiden, ob es sich (noch immer) um eine eigenständige Organisation, um ein Netzwerk, oder um eine Organisation als fokalen Akteur ( $h u b$ oder „Nabe“ ) in einem Netzwerk handelt. 4

\section{Hybridstrukturen zwischen Hierarchie und Netzwerk}

Eine hierarchische Struktur gilt allgemein als kennzeichnend für den ,alten" nationalen Terrorismus. Einzelne Fallstudien nationaler Terrororganisationen - der ETA (u.a. Iribarren 1998), IRA (u.a. Horgan/Taylor 1997), SL (u.a. McCormick 1990), Hamas (u.a.
Ranstorp 1994) - beschreiben diese hierarchischen Strukturen recht genau. Die Literatur zum ,neuen", inter- oder transnationalen Terrorismus betont dagegen seine Netzwerkstruktur (so u.a. Hoffmann 1999; Simon/Benjamin 2000; Hirschmann 2000; Roth 2001; Arquilla/Ronfeldt 2001; Gunaratna 2002; Rothenberg 2002; Schneckener 2002), wobei, wie schon gesagt, nicht zwischen Netzwerk als einem bestimmt strukturierten sozialen Gebilde und Netzwerk als analytischem Merkmal unterschieden wird. Diese begriffliche Mehrdeutigkeit hängt mit den Unschärfen der geläufigen Typen sozialer Handlungskoordination zusammen. In der Trias Markt, Hierarchie und Netzwerk (vgl. Thompson et al. 1991) werden Organisationen leicht dem Typ Hierarchie zugeordnet, am deutlichsten dort, wo Firmen, wie bei Williamson (1975), Märkten gegenübergestellt und als „Hierarchie" bezeichnet werden. Organisationssoziologisch betrachtet sind Organisationen jedoch zwar ex definitione (auch) vertikal differenziert, müssen aber - man denke an die große Kategorie demokratisch verfasster Organisationen - nicht zugleich "hierarchisch" im Sinne von zentral und top-down gesteuert sein. Gerade die Organisationssoziologie hat sich ganz im Gegenteil darum bemüht, die Unterschiedlichkeit der Struktur verschiedener Organisationen herauszuarbeiten und dabei unter anderem mit dem Modell der professionellen oder ,organischen" Organisation (Burns/ Stalker 1961) einen Gegentypus zum hierarchisch-bürokratischen Modell entwickelt, der erkennbar mit dem verwandt ist, was heute als netzwerkartige Organisation beschrieben wird.

Während der ,alte“, nationale Ziele verfolgende Terrorismus am Modell des Militärs orientiert war oder ist, orientiert sich der "neue" internationale Terrorismus eher am Modell eines durch Netzwerkmerkmale gekennzeichneten multinationalen Untermehmens (vgl. Bergen 2001: 43ff.; Heiligsetzer 2002: 153 spricht sogar von der „Terroris-

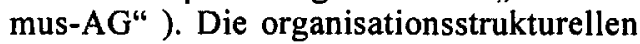
Unterschiede zwischen altem und neuem Terrorismus sind jedoch gradueller Natur. Tatsächlich weisen alle verdeckt operierenden Terrororganisationen eine Kombination 
von hierarchischen und Netzwerkmerkmalen auf. So charakterisieren z.B. Horgan und Taylor die IRA explizit als eine Hybridform von „Organisation“" und „Netzwerk" , ,a cellular-based, hierarchically-organized authoritarian structure" (Horgan/Taylor 1997: 3). Diese Mischung aus einer netzwerkartigen Zellstruktur und zentraler Steuerung findet sich in allen verfügbaren Strukturbeschreibungen des organisierten Terrorismus, gleich ob es sich um Organisationen mit nationalem Zielbezug oder Al Qaida handelt. Zwar wird gelegentlich betont, dass islamische Organisationen sich zutreffender als "ringförmig“" aufgebaut beschreiben lassen ("Islamic organizations are better thought of as building from an internal core outward"; DSSI 2001: 8). Aber aus der konkreten Beschreibung einzelner islamischer Organisationen ergibt sich, dass auch sie sowohl hierarchische wie Netzwerkmerkmale haben.

Übereinstimmend finden sich in den Strukturbeschreibungen aller hier betrachteter terroristischer Organisationen und von $\mathrm{Al}$ Qaida die folgenden Hierarchiemerkmale:

- Es gibt klar definierte Führungsgremien (die cupola bei SL, die Army Executive bei der IRA, majlis shura beim islamischen Jihad, die manchmal als council bezeichnete Führungsgruppe bei Al Qaida).

- Es besteht eine erkennbare Differenzierung nach Rängen und nach Funktionen. ${ }^{5}$ Zum einen haben ausnahmslos alle nationalen Organisationen ebenso wie Al Qaida unterhalb der Leitung spezialisierte Einheiten unter anderem für Beschaffung, Finanzierung, Ausbildung, Nachrichtenwesen und Öffentlichkeitsarbeit. Sie sind entweder in Form einer Matrixorganisation mit den Einheiten der nächst folgenden Ebene verbunden, gelegentlich aber auch in Form von zwei getrennten Zweigen, einem „militärischen" und einem „logistisch/ unterstïtzenden", organisiert. Auf der mittleren Ebene gibt es außerdem Personen, die die Verbindung zwischen der Fuhrungsgruppe und den ausführenden Einheiten halten, dabei aber uber eigene Befehlsgewalt verfugen. Ausnahmslos in allen Formen des organisierten Terrorismus gibt es schließlich eine klar abgegrenzte untere Ebene von operativen, ausfuhrenden Einheiten.

- Es dominiert die vertikale Kommunikation.
Neben diesen hierarchietypischen Merkmalen finden sich in allen hier behandelten Terrororganisationen auch die folgenden, für Netzwerke charakteristischen Merkmale:

- Es findet keine zentrale Detailsteuerung des Handelns statt. Die Untereinheiten, vor allem aber die häufig als „Zellen" (IRA: Active Service Units - ASU) bezeichneten operativen Einheiten auf unterster Ebene, sind in ihrem Handeln relativ autonom. Eine solche Teil-Autonomie entspricht dem islamischen Unqud oder Weintraubenmodell (Heiligsetzer 2002: 153). Zellen werden durchweg als klein und intern differenziert beschrieben.

- Die Organisation hat eine relativ offene und zugleich fließende Grenze. Zum einen werden ständig Zellen aufgelöst und treten neu hinzu. Zum anderen gibt es Mitglieder verschiedenen Grades in Bezug auf ihre Fremd- und Selbstidentifikation, d.h. Mitgliedschaft ist eine Variable: Es gibt Aktivisten (core members, Berufsterroristen), die im Extremfall keine Exit-Option haben; Mitglieder, die bestimmte Aufgaben erfullen, zugleich aber ein normales Leben in der legalen Welt führen; und verlässliche Helfer, auf die fallweise zurückgegriffen wird und die oft nicht einmal wissen, für wen und wozu sie Hilfe leisten.

- Die Organisation reagiert durch schnelles Verwerfen bestehender und Entwerfen neuer Pläne und durch Veränderungen in Funktion und Positionierung von Personen und Einheiten flexibel auf Veränderungen (Chancen, Bedrohungen) in den äußeren Umständen. Das Ergebnis ist eine gewisse Fluidităt der Struktur, was von normalem, geplanten Organisationswandel deutlich zu unterscheiden ist.

Zwar gibt es zwischen den verschiedenen hier behandelten Manifestationen des organisierten Terrorismus deutliche Unterschiede im relativen Gewicht entweder hierarchieoder netzwerktypischer Merkmale, doch weisen sie alle tatsächlich die genannten Merkmale beider Formen auf. Eine solche Merkmalskombination erscheint widerspruchlich, was in der Literatur auch gelegentlich bemerkt wird. So stellt etwa Richard Rothenberg (2002) fest, im organisierten Terrorismus würden eigentlich einander widerspre- 
chende Elemente miteinander kombiniert: die Dominanz horizontaler Differenzierung, aber dennoch klare Führer; das Bemuhen um die Sicherung der Organisationsgrenze, aber trotzdem eine dynamische Bildung neuer Zellen. Das Ergebnis einer solchen Merkmalskombination ist eine organisatorische Hybridstruktur. Hybridstrukturen sind im Bereich von Organisationen keine Seltenheit ${ }^{6}$ und gelten auch keineswegs als grundsätzlich disfunktional. Im Fall von Terrororganisationen wird als wesentlicher Grund für die Beimischung von Netzwerkmerkmalen zu einer - Kampforganisationen angemessenen - hierarchischen Struktur gewöhnlich die besondere Situation ihres Wirkens im Untergrund genannt, die Notwendigkeit, die Unversehrtheit der Organisation zu gewährleisten und dennoch gleichzeitig ihre Effektivität zu sichern. Dieser kausal-genetische Zusammenhang ist gut am Beispiel der IRA nachzuvollziehen, die in Reaktion auf zunehmend erfolgreiche Repression ihre zunächst stärker am militärischen Modell orientierte Organisation in Richtung eines Netzwerkmodells modifizierte (Horgan/Taylor 1997).

Die Organisation des (uber das Niveau von isolierten Kleingruppen hinausgehenden) Terrorismus lässt sich allerdings nur unzulänglich als Mischform aus den genannten hierarchischen und Netzwerkmerkmalen beschreiben. Gleichgültig, ob wir es (vom Gebildetypus her) mit terroristischen Organisationen oder einem Netzwerk zu tun haben und ob eine Organisation im Einzelfall stärker hierarchisch geprägt oder durch Netzwerkmerkmale gekennzeichnet ist, finden wir durchweg eine Reihe zusätzlicher Merkmale, die weder ins hierarchische Muster passen noch allgemein netzwerktypisch sind. Die Identifikation dieser Merkmale ist das wichtigste Ergebnis der hier angestellten Analyse von Organisationsformen des Terrorismus.

An erster Stelle zu nennen ist die Tatsache, dass das Handeln der Einheiten auf den nachgeordneten Rängen, bei aller Autonomie, über die sie im Hinblick auf ihre konkreten Aktivitäten verfügen, doch zugleich einer zentralen Steuerung unterliegt, und gemein formulierter Ziele und Strategien. Sofern konkrete Weisungen gegeben werden, sind diese meist fallbezogen (ad hoc) und nur an konkrete Einzelpersonen gerichtet.

Das zweite Merkmal ist ein besonderer Modus loser Kopplung zwischen den einzelnen Einheiten, der durch eine weitgehende Latenz vertikaler und horizontaler Beziehungen gekennzeichnet ist. „Latente“ Beziehungen sind zwar (wie eine fleet in being) vorhanden, manifestieren sich aber nicht in ständigen (beobachtbaren) Interaktionen. Die face-to-face Kommunikation ist vielmehr sowohl in vertikaler wie in horizontaler Richtung stark eingeschränkt. Besonders die untersten Einheiten (Zellen) sind kommunikativ gegeneinander isoliert. ${ }^{7}$ Hinzu kommt eine bewusst inszenierte Intransparenz der Organisation fur die operativen Einheiten. Die dennoch latent immer vorhandenen $\mathrm{Be}-$ ziehungen lassen sich fallweise, "bei Bedarf“" (Bendel/Hildebrandt 2002: 19) aktivieren. Krebs (2002) beschreibt ganz in diesem Sinne das durch "transitory short-cuts" aktivierte, unsichtbare Beziehungsnetz der am Anschlag vom 11. September beteiligten Terroristen.

Diese beiden „besonderen“" Merkmale terroristischer Organisation stützen sich auf und werden ermöglicht durch ein drittes Merkmal, nämlich eine starke, durch Sozialisation (ideologische Indoktrination und praktisches Lernen) bewirkte Identifikation der Mitglieder mit der Organisation und ihren Zielen, ergänzt und stabilisiert durch enge persönliche Beziehungen insbesondere zwischen den Mitgliedern der Führungsgruppe und zwischen den aktiven Mitgliedern von operativen Zellen. Auf dieser Art der Bindung beruht die Möglichkeit einer gewissermaßen auf die „Richtlinien der Politik" beschränkten und dennoch wirksamen Steuerung ebenso wie die Existenz jederzeit aktivierbarer latenter Beziehungen. Als eine Form von Kopplung verschiedener Untereinheiten oder Netzwerkknoten setzen latente Beziehungen eine grundsätzliche Übereinstimmung und gewissermaßen "blindes" Vertrauen voraus; ohne sie wäre eine Beziehung bei fehlender kontinuierlicher Interaktion nicht problemlos aktivierbar. ${ }^{8}$ In diesem Zusammenhang mag einer tief empfundenen religiösen Überzeu- 
gung als Basis von Identifikation und Vertrauen eine besondere Bedeutung zukommen.

Die drei soeben beschriebenen Merkmale ${ }^{9}$ treten in terroristischen Organisationen immer gemeinsam auf und bilden insofern ein einziges, eng verknüpftes Cluster. Weder der besondere Steuerungsmechanismus noch die Kopplung durch latente Beziehungen gehören als Merkmal zu einem der gewöhnlich unterschiedenen Typen nicht-marktlicher Handlungskoordination (Hierarchie, Gemeinschaft, Netzwerk). Zentrale Steuerung durch die Vorgabe allgemein formulierter Ziele und Strategien gehört weder zum Idealtypus Hierarchie noch zum Netzwerk. In Netzwerken beruht die Handlungskoordination auf Verhandlung und Austausch, in hierarchischen Organisationen auf einem ständjgen Strom expliziter Weisungen oder auf einem ausgefeilten System von Verhaltensregeln, die für die Inhaber bestimmter Positionen gelten. Verhandeln impliziert ebenso wie Anweisen manifeste Interaktionen. Das Cluster der ,besonderen“" Merkmale terroristischer Organisation lässt sich auch nicht dem Typus der solidarischen Gemeinschaft zuordnen. Eine starke Identifikation und enge persönliche Beziehungen sind zwar „gemeinschaftstypisch", aber Gemeinschaften sind durch manifeste Beziehungen und das Fehlen jeglicher Art zentraler Steuerung gekennzeichnet.

Die „besonderen“", d.h. weder hierarchienoch netzwerk- oder gemeinschaftstypischen Merkmale können Überleben und Effektivität einer terroristischen Organisation bzw. eines Netzwerks sichern helfen. Die Beimischung "einfacher" Netzwerkmerkmale genügt dafür offenbar nicht. Latente Beziehungen schutzen die Organisation vor Entdeckung und Aufrollen, während der besondere Steuerungsmodus verhindert, dass die in einer bedrohten Situation unerlässliche Autonomie von Untereinheiten die Organisation von innen heraus desintegrieren oder ineffizient werden lässt. Diese besonderen Merkmale terroristischer Organisation finden sich auch in nationalen Terrororganisationen wie u.a. dem Leuchtenden Pfad (SL); ihre funktionelle Bedeutung wächst jedoch mit der internationalen Reichweite und wird um so größer, je mehr wir uns dabei vom Gebildetyp der Organisation fort und hin zum (interorganisationalen) terroristischen Netzwerk bewegen.

Die idealtypische Beschreibung der „besonderen" Merkmale terroristischer Organisation und der Hinweis auf ihre Funktionalităt sollten nicht zu der Annahme verführen, sie seien tatsächlich stets in der erforderlichen Ausprăgung vorhanden. Im Gegenteil: Der organisierte Terrorismus ist nicht nur durch Repression und die Erosion externer Unterstützung, sondern auch durch endogene Zerfallstendenzen in seinem jeweiligen Bestand gefăhrdet. Dazu gehören besonders in eher hierarchisch integrierten Organisationen ideologische und strategische Auseinandersetzungen, die die Grundlage der gemeinsamen Identifikation und damit auch die Steuerbarkeit der Organisation gefährden. Aber auch die Netzwerkorganisation ist stets vom Zerfall bedroht. Diesen Zerfall beschreibt Frisch (1991) eindrucksvoll am Beispiel der palästinensischen (ersten) Intifada. Das selbstständige und doch zielgerechte Handeln überwiegend latent miteinander verbundener Einheiten ist ein immanent instabiler Modus von Organisation.

\section{Ergebnisse und offene Fragen}

Die in diesem Aufsatz herausgearbeiteten Besonderheiten terroristischer Organisation sind nicht nur von phänomenologisch-deskriptivem Interesse. Sie können in der Terrorismusforschung zu einer Differenzierung der holzschnittartigen Gegenüberstellung von ,alten“ hierarchischen und "neuen" netzwerkartigen Organisationsformen des Terrorismus dienen. Auch ,alte" nationale Terrororganisationen haben Netzwerkmerkmale, und sie können außerdem zu ihrer Unterstützung ein internationales Netzwerk aufbauen, in dem sie die Rolle der fokalen Organisation spielen. Diese internationalen Netzwerke sind zu unterscheiden von transnationalen terroristischen Netzwerken, die keine nationalen Ziele mehr verfolgen und deren Mitglieder über mehrere Länder verstreute, intern sehr unterschiedlich struk- 
turierte lokale Gruppen und Organisationen sind; dieser Strukturtyp ist heute mit dem Namen Al Qaida verbunden. Auch in solchen Netzwerken mag es einen zentralen Netzwerkknoten geben. Im Unterschied zu einem der Unterstützung einer nationalen Organisation dienenden internationalen Netzwerk sind die einzelnen „Knoten“ im transnationalen Netzwerk jedoch nicht durch einfache Hilfs- oder Zuliefererfunktionen mit der fokalen Organisation verbunden, sondern besitzen ein hohes Maß an (funktioneller) Eigenständigkeit.

Die verschiedenen hier angesprochenen Netzwerkarten verdienten eine sehr viel genauere Beschreibung und Kategorisierung, als infolge mangelnder Detailinformation möglich ist. In der Literatur wird vor allem mit Bezug auf Al Qaida gelegentlich versucht, bekannte Grundformen von Netzwerken wie Kette (chain), Rad oder Stern ( $h u b$ \& spoke, star) und vollständig verbundene Graphen (all-channel) für die Strukturbeschreibung zu nutzen (z.B. Arquilla/Ronfeld 2001: $7 \mathrm{ff}$;; Simon/Benjamin 2000: 70), was jedoch bislang wenig mehr als den Hinweis erbracht hat, dass in der Realität terroristischer Organisation alle diese Formen kombiniert vorkommen. Derartige Kombinationen lassen sich wohl schematisch darstellen (vgl. Carley/Lee/Knackhardt 2002), genügen aber kaum zur Erfassung der realen Komplexität terroristischer Netzwerke. Vor allem latente Beziehungen lassen sich nicht ohne weiteres mit den Mitteln formaler Netzwerkanalyse abbilden. ${ }^{10}$ Die formale Netzwerkanalyse steht hier noch vor großen Herausforderungen.

Das wichtigste Ergebnis der vorstehenden Analyse - die Identifikation der drei zusammenhăngenden, besonderen Merkmale terroristischer Organisation - leistet einen Beitrag zur Theorie der Handlungskoordination speziell in interorganisatorischen Netzwerken. Derartige Netzwerke spielen sowohl auf nationaler wie zunehmend auf internationaler bzw. "globaler" Ebene eine wichtige Rolle, in der Ökonomie in Form verschiedener Arten von Unternehmensnetzwerken (Allianzen, Kartellen, production networks) und in der Politik in Form von Politiknetzwerken
Allen Netzwerken gemeinsam ist das Problem der Koordination, auf das jedoch offensichtlich durchaus unterschiedliche Antworten gefunden werden. Gewöhnlich werden Organisationsnetzwerke in steuerungstheoretischer Perspektive als Verhandlungssysteme korporativer Akteure betrachtet, die im wechselseitigen Austausch von Argumenten, Angeboten und Drohungen (z.B. mit Unterstützungsentzug) eine gemeinsame Entscheidung suchen und bei entsprechender Ressourcenverteilung und Interessenstruktur auch finden. Auf Unternehmensnetzwerke passt dieses Konzept weniger gut als auf Politiknetzwerke"ll und auf die Koordination im organisierten Terrorismus uberhaupt nicht. Hier beruht die Handlungskoordination vielmehr im Wesentlichen auf dem beschriebenen besonderen Steuerungsmechanismus, d.h. auf der - aus einem hohen (und wahrscheinlich emotional aufgeladenen) ideologischen Konsens resultierenden - bereitwilligen Akzeptanz der von anerkannten, aber über keine formale Autorităt verfügenden Führungspersonen ausgehenden Signale. Gewissermaßen als Nervenstränge sind dabei in die nur locker gekoppelte Struktur persönliche Beziehungsnetze eingelagert, die - anders als bei den interpersonell verflochtenen Unternehmensnetzwerken - auf Verwandtschaft, gemeinsamer ethnischer Zugehörigkeit und gemeinsamen Erfahrungen (z.B. in Trainingscamps) aufbauen und zur reibungslosen Übermittlung von handlungsleitenden Impulsen beitragen.

Haben wir es also, um zu der eingangs gestellten Frage zurückzukehren, beim organisierten Terrorismus mit einer Organisationsform sui generis zu tun? Sicher nicht aufgrund der Tatsache, dass "alter" wie "neuer" Terrorismus durch eine Mischung von Merkmalen hierarchischer und netzwerkartiger Organisation gekennzeichnet ist. Bei näherem Hinsehen sind aber auch die einzelnen Merkmale aus dem - keinem der geläufigen Typen sozialer Handlungskoordination zuordenbaren - Cluster "besonderer" Merkmale nicht nur im organisierten Terrorismus zu finden. Eine ideologisch basierte Identifikation findet sich auch anderswo, nicht nur in Sekten und sozialen Bewegungen, sondern auch in manchen (legalen) Kampfverbänden 
und sogar in Unternehmen. Auch latente Beziehungen finden sich nicht nur im organisierten Terrorismus; sie sind generell eine wichtige Voraussetzung erfolgreicher Klandestinität, ob es sich dabei um Geheimbünde, organisierte Kriminalität oder die Schattenwirtschaft handelt. Das Besondere am organisierten Terrorismus ist eher der mit starker Identifikation und Latenz von Beziehungen verknüpfte Modus der Steuerung über allgemeine Ziel- und Strategievorgaben, also das Cluster der drei Merkmale, die zusammen eine besondere Form der Handlungskoordination jenseits von hierarchischer Weisung, Verhandlung und gemeinschaftlicher Befolgung von Gruppennormen ausmachen.

Diese Form der Handlungskoordination stellt einen selten als solchen beschriebenen Typus dar, der jedoch nicht strikt an eine terroristische Zielsetzung und das Leben im Untergrund gebunden scheint. Die beschriebene Form der Handlungskoordination findet sich ahnlich in dem von Udo Ulfkotte (2003) detailreich analysierten Netz international verflochtener, nicht-terroristischer und formal legaler islamistischer Gruppen und Einrichtungen in Deutschland. Auch hier werden die verschiedenen, religiös gleichgerichteten Vereine, Zentren und Organisationen durch interpersonelle Beziehungen zusammengehalten, die in die interorganisatorischen $\mathrm{Be}-$ ziehungen eingebettet sind. Obwohl es (in Gestalt von Milli Görus) einen Netzwerkknoten von hoher Zentralität gibt, herrscht verteilte horizontale Einflussnahme ohne autoritative Steuerung vor. Bei dieser Art der spontanen und dennoch zugleich zielgerichteten Handlungskoordination werden Steuerungsimpulse eher nach dem Muster des für neuronale Netze typischen parallelen Prozessierens an vielen Punkten gleichzeitig generiert. Die keiner von außen erkennbaren Regel folgende Aktivierung einzelner Verbindungen und Einheiten erzeugt dabei den Eindruck einer pulsierenden Organisation, einer Organisation, die zwischen verschiedenen Strukturmustern oszilliert, mal stärker zentral gesteuert, mal dezentral handelnd. Das erinnert an die für den Außenstehenden schwer begreifbare Funktionsweise der Organisation auf einem amerikanischen Flugzeugträger, die Weick und Roberts
(1993) geschildert haben. Eine nähere Erkundung dieser verwandten Formen von Handlungskoordination, die einen eigenen, noch der semantischen Taufe harrenden Typus bilden, wäre sicher wünschenswert.

Einsichten in die Besonderheiten terroristischer Organisation sind auch anschlussfähig zu anderen Feldern sozialwissenschaftlicher Theoriebildung; auf einige dieser Anschlussmöglichkeiten sei abschließend kurz hingewiesen. Der sich andeutende $\mathrm{Zu}$ sammenhang zwischen den Überlebensbedingungen terroristischer Organisationen und ihren Strukturmerkmalen könnte zur Erweiterung des kontingenztheoretischen bzw. ,situativen" Ansatzes dienen, in dessen Zentrum der Zusammenhang zwischen Umweltmerkmalen und der Struktur ökonomischer Organisationen steht (vgl. Lawrence/Lorsch 1967; Kieser/Kubicek 1983: 317ff.). Zu fragen wäre, wie weit sich die Schlussfolgerungen im Hinblick auf die strukturelle Anpassung von zielgerichtet handelnden Organisationen an die Überlebensbedingungen einer bedrohten Untergrundexistenz auf andere als terroristische Ziele verfolgende, aber gleichwohl klandestine Organisationen ubertragen lassen. Hier wäre ein Vergleich mit der organisierten Kriminalität lehrreich.

In dynamischer Perspektive könnten die verfügbaren Beschreibungen der Entwicklungsgeschichte einzelner terroristischer Organisationen Anlass sein, nach der - wohl im Fall von Al Qaida besonders ausgeprägten - historischen „Pfadabhängigkeit" ihrer jeweils vorfindbaren Struktur zu fragen. Die Gegenüberstellung von historisch-genetischen und auf den Struktur/Umwelt-Nexus konzentrierten Erklärungsansätzen fuhrt dabei zu der für die Theorie institutionellen Wandels zentralen Frage, ob der bisherige Entwicklungspfad oder die aktuelle Situation eine Organisation stärker prägen. Die phasenweise bei der ETA und IRA beobachtete Radikalisierung als Überlebensstrategie der Organisation angesichts nachlassender Unterstultzung durch die Bevölkerung bzw. nachlassenden Repressionsdruckes illustriert sehr gut, wie in bestimmten Situationen beides zusammenwirkt. So könnte die Ausdehnung der vergleichenden Sozialforschung auf Organisationen von der „Nachtseite" un- 
serer Welt in verschiedenen Bereichen der Theoriebildung horizonterweiternd sein.

\section{Anmerkungen}

$1 \mathrm{Zu}$ der - problematischen und in der Literatur vielfach erörterten - Definition von Terrorismus hier nur kurz der Hinweis auf die zwei zentralen Kriterien Zielrichtung und Strategie. Das Ziel terroristischer Gruppierungen ist ein politisches: Es geht um die grundlegende Veränderung eines Status quo, und zwar nicht durch legale Ausübung von "voice", sondern durch die destabilisierende Schockwirkung unvorhersehbarer Gewalttaten gegen nicht an einer offenen Auseinandersetzung Beteiligte, d.h. meist Zivilisten. Terroristische Organisationen unterscheiden sich von Guerillaorganisationen durch ihre Strategie und von der sogenannten organisierten Kriminalităt durch ihr politisches (anstatt ökonomisches) Ziel. Illegalität (aus der Perspektive der je herrschenden Ordnung) ist kein zentrales Merkmal von Organisationen, die Terrorismus als Strategie einsetzen. Obwohl sie mindestens dort klandestin arbeiten, wo Sanktionen zu fürchten sind, sind Organisationen wie die Hamas oder die IRA als solche bekannt; ihre Mitglieder bekennen sich zum Teil offen zu ihnen und ihre Führungspersonen sind (zumindest teilweise) namentlich bekannt.

2 Organisationen sind durch die Existenz einer "Mitgliedschaftsrolle" nach außen klar abgegrenzt und besitzen eine vertikal und funktionell differenzierte Rollenstruktur, die von den konkreten Rolleninhabern unabhängig ist; in Netzwerken sind dagegen selbstständige, konkrete Einheiten (Personen, Gruppen, Organisationen) interaktiv verknüpft.

3 Auf die interessante Frage, wieweit das Entstehen von Al Qaida auf einer ganz besonderen historischen Konstellation (sowjetische Invasion von Afghanistan, Kalter Krieg, Existenz eines Unternehmers wie Bin Laden) beruht und wieweit auf Faktoren, die ganz allgemein den internationalen Terrorismus begünstigen, sei hier nur am Rande verwiesen.

4 Dies vermutet z.B. Behr (2002: $111 \mathrm{ff}$ ); Elwert (2002) behandelt Al Qaida einmal als Netzwerk (125) und dann wieder als Organisation in einem Netzwerk (127). Tatsächlich ist nicht grundsătzlich auszuschließen, dass der verbrejtete Eindruck, es handele sich bei Al Qaida um ein Netzwerk, lediglich die Folge unvollständiger Information über die Struktur einer verdeckt existierenden, integrierten Organisation ist. mistischen Terrororganisationen zu einer Art von Doppelhierarchie, wie sie aus EinparteienDiktaturen bekannt ist, in denen es parallele und auf jeder Ebene miteinander verknüpfte Hierarchien von Staat und Partei gibt. In islamistischen Terrororganisationen entsteht eine solche Struktur durch die Doppelung zwischen Geistlichen (Mullahs etc.) und militärischen bzw. (natürlich ebenfalls gläubigen) „welttlchen" Kadern. Ranstorp (1994) hat diese Struktur ausführlich für die Hizbollah beschrieben.

6 So sind internationale Organisationen wie die WTO, der IWF oder die ILO als Hybridform von Organisation und Netzwerk beschrieben worden (Mayntz 2002).

7 Vgl. hierzu Heiligsetzer (2002: 154): „Aus Sicherheitsgründen existieren vertikal zwischen den Ebenen und auch horizontal zwischen den einzelnen Zellen (...) zahlreiche Barrieren. Zwar kennen die höheren Chargen die jeweils unteren, aber Anweisungen werden trotzdem selten persönlich gegeben (...) Innerhalb des militärischen Flügels (von Hamas; RM) kennt in der Regel kein einziges Mitglied die Angehörigen der zweithöheren Ebene".

8 "Shared values" sind in der Literatur als Kopplungsmechanismus durchaus geläufig; dagegen fehlt offenbar in der Literatur zur lockeren Kopplung (loose coupling) der Hinweis auf den hier beschriebenen Modus der über Konsens vermittelten latenten Beziehungen. $\mathrm{Vgl}$. hierzu den Übersichtsaufsatz von Orton und Weick (1990).

9 Die einzelnen dieser besonderen Merkmale werden in der Literatur teils phänomenologisch beschreibend dargestellt, teils explizit genannt; das gilt vor allem für die Bedeutung der starken, ideologisch basierten Identifikation. $\mathrm{Vgl}$. unter anderem Schneckener (2002: 23), der auch andere der hier beschriebenen Merkmale erwăhnt. Besonders ausführlich werden die Besonderheiten terroristischer Organisation von Arquilla und Ronfeldt (2001) behandelt.

10 Das soll nicht heißen, dass das prinzipiell unmöglich ist; Verspagen/Werker (2003) haben z.B. latente, d.h. nicht über Interaktionen laufende Beziehungen in einer scientific community dargestellt.

11 Zur Koordination in internationalen Netzwerken kleiner und mittlerer Unternehmen vgl. z.B. Hirsch-Kreinsen/Wannơffel (2003). 


\section{Literatur}

Abanes, Richard (1996): American Militias. Downer's Grove, Ill.: InterVarsity.

Arquilla, John/David Ronfeldt (2001): The Advent of Netwar (Revisited). In: John Arquilla/ David Ronfeldt (Hrsg.), Networks and Netwar. The Future of Terror, Crime, and Militancy. Santa Monica, RAND Corporation, S. 1-25.

Bartlett, Christopher A./Goshal Sumantra (1989): Managing Across Borders. The Transnational Solution. Boston: Harvard Business School Press.

Behr, Hartmut (2002): Neue Organisationsformen des Terrorismus und Ordnungstypologien transnationaler Politik. In: Petra Bendel/ Mathias Hildebrand (Hrsg.), Im Schatten des Terrorismus. Hintergründe, Strukturen, Konsequenzen des 11. September 2001. Wiesbaden: Westdeutscher Verlag, S. 109-130.

Bendel, Petra/Mathias Hildebrandt (2002): Der 11. September: Hintergründe, Strukturen und Konsequenzen in der wissenschaftlichen und politischen Diskussion. In: Petra Bendel/ Mathias Hildebrand (Hrsg.), Im Schatten des Terrorismus. Hintergründe, Strukturen, Konsequenzen des 11. September 2001. Wiesbaden: Westdeutscher Verlag, S. 9-32.

Bergen, Peter L. (2001): Holy War, Inc. Inside the Secret World of Osama Bin Laden. London: Weidenfeld \& Nicolson.

Burns, Tom/George M. Stalker (1961): The Management of Innovation. London: Tavistock Publications.

Carley, Kathleen M./Ju-Sung Lee/David Krackhardt (2002): Destabilizing Networks. In: Connections 24, S. 79-91.

Dekmejian, R. Hrair (1995): Islam in Revolution. Fundamentalism in the Islamic World. Syracuse, N.Y.: Syracuse University Press.

Della Porta, Donatella (1995): Social Movements, Political Violence, and the State. A Comparative Analysis of Italy and Germany. Cambridge: Cambridge University Press.

DSSI/Decision Support Systems Inc. (2001): Hunting the Sleepers. Tracking Al-Qaida's Covert Operatives. Computer-Ausdruck/ Electronic Source.

Elwert, Georg (2002): Weder irrational noch traditionalistisch. Charismatische Mobilisierung und Gewaltmärkte als Basis der Attentäter des 11. September. In: Hauser-Schäublin, Brigitta/Ulrich Braukämper (Hrsg.), Ethnologie der Globalisierung - Perspektiven kultureller Verflechtungen. Berlin: Reimer, S. 125-151.

Frisch, Hillel (1991): Between Diffusion and Territorial Consolidation in Rebellion: Striking at the Hard Core of the Intifada. In: Terrorism and Political Violence 3, S. 39-62.

Gunaratna, Rohan (1998): International and Regional Implications of the Sri Lankan Tamil Insurgency. International Policy Institute for Counter Terrorism: Electronic Source Download.

Gunaratna, Rohan (2002): Inside Al Qaeda. Global Network of Terror. London: Hurst.

Heiligsetzer, Edda (2002): Extremismus, Terrorismus, „Heiliger Krieg“" : Zur Soziologie religiöser Terroristen. In: Petra Bendel/Mathias Hildebrand (Hrsg.), Im Schatten des Terrorismus. Hintergründe, Strukturen, Konsequenzen des 11. September 2001. Wiesbaden: Westdeutscher Verlag, S. 150-167.

Hirsch-Kreinsen, Hartmut/Manfred Wannoffel (Hrsg.) (2003): Netzwerke kleiner Unternehmen - Praktiken und Besonderheiten der internationalen Zusammenarbeit. Berlin: edition sigma.

Hirschmann, Kai (2000): The Changing Face of Terrorism. In: International Politics and Society 7, S. 299-310.

Hoffmann, Bruce (1999): Terrorism. Trends and Prospects. In: Ian O. Lesser et al. (Hrsg.), Countering the New Terrorism. Santa Monica, Calif.: RAND, S. 7-38.

Hollingsworth, J. Rogers/Philippe C. Schmitter/Wolfgang Streeck (Hrsg.) (1994): Governing Capitalist Economies: Performance and Control of Economic Sectors. New York: Oxford University Press.

Horgan, John/Max Taylor (1997): The Provisional Irish Republican Army: Command and Functional Structure. In: Terrorism and Political Violence 9, S. 1-32.

Iribarren, Florencio Domínguez (1998): ETA: Estrategia Organizativa y Actuaciones 19781992. Bilbao: Servicio Editorial Universidad del Pais Vasco.

Kieser, Alfred/Herbert Kubicek (1983): Organisation. Berlin/New York: de Gruyter.

Kitschelt, Herbert (2003): State Failure, Globalization, and Regime Conflict: Origins of Contemporary International Terrorism in the Middle East. Friedrich Ebert Stiftung, Reihe Internationale Politikanalyse.

Krebs, Valdis E. (2002): Mapping Networks of Terrorist Cells. In: Connections 24, S. 43-52.

Laqueur, Walter (1999): The New Terrorism. Oxford: Oxford University Press.

Lawrence, Paul R./Jay W. Lorsch (1967): Organization and Environment. Boston, Mass.: Harvard University Press.

Marin, Bernd/Renate Mayntz (Hrsg.) (1991): Policy Networks. Empirical Evidence and 
Theoretical Considerations. Frankfurt a.M.: Campus.

Mayntz, Renate (1963): Soziologie der Organisation. Reinbek bei Hamburg: Rowohlt.

Mayntz, Renate (1993): Modernization and the Logic of Interorganizational Networks. In: John Child et al. (Hrsg.), Societal Change Between Market and Organization. Aldershot, UK u.a.: Avebury, S. 3-18.

Mayntz, Renate (2002): Internationale Organisationen im Prozess der Globalisierung. In: Peter Nahamowitz/Rudiger Voigt (Hrsg.), Globalisierung des Rechts II: Internationale Organisationen und Regelungsbereiche. BadenBaden: Nomos, S. 85-100.

McCormick/Gordon H. (1990): The Shining Path and the Future of Peru. RAND report R-3781DOS/OSD.

Mishal, Shaul/Avraham Sela (2000): The Palestinian Hamas. New York: Columbia University Press.

Münkler, Herfried (1992): Gewalt und Ordnung. Das Bild des Krieges im politischen Denken. Frankfurt a.M.: Fischer.

Münkler, Herfried (2001): Sind wir im Krieg? Über Terrorismus, Partisanen und die neuen Formen des Krieges. Politische Vierteljahresschrift 42, S. 581-589.

Neidhardt, Friedhelm (1982): Linker und rechter Terrorismus. Erscheinungsformen und Handlungspotentiale im Gruppenvergleich. In: Wanda von Baeyer-Katte/Dieter Claessens/ Hubert Feger/Friedhelm Neidhardt (Hrsg.), Gruppenprozesse. Opladen: Westdeutscher Verlag, S. 433-476.

Orton, J. Douglas/Karl E. Weick (1990): Loosely Coupled Systems: A Reconceptualization. In: Academy of Management Review 15, S. 203223.

Ranstorp, Magnus (1994): Hizbollah's Command Leadership: Its Structure, Decision-Making and Relationship with Iranian Clergy and Institutions. In: Terrorism and Political Violence 6, S. 303-339.

Reich, Walter (Hrsg.) (1990): Origins of Terrorism: Psychologies, Ideologies, Theologies, States of Mind. Cambridge: Woodrow Wilson Center \& Cambridge University Press.

Reinicke, Wolfgang H./Jan Martin Witte (1999): Globalization and Democratic Governance: Global Public Policy and Trisectoral Networks. In: Carl Lankowski (Hrsg.), Governing
Beyond the Nation-State. Global Public Policy, Regionalism, or Going Local? Washington, D.C.: American Institute for Contemporary German Studies, S. 1-39.

Roth, Jürgen (2001): Netzwerke des Terrors. Hamburg/Wien: Europa Verlag.

Rothenberg, Richard (2002): From Whole Cloth: Making up the Terrorist Network. In: Connections 24, S. 36-42.

Schneckener, Ulrich (2002): Netzwerke des Terrors. Charakter und Strukturen des transnationalen Terrorismus. SWP-Studie S 42. Berlin: Stiftung Wissenschaft und Politik.

Schröm, Oliver (2002): Im Schatten des Schakals. Carlos und die Wegbereiter des internationalen Terrorismus. Berlin: Ch. Links Verlag.

Simon, Steven/Daniel Benjamin (2000): America and the New Terrorism. In: Survival/IISS Quarterly 42, S. 59-75.

Straßner, Alexander (2003): Die dritte Generation der "Roten Armee Fraktion" . Entstehung, Struktur, Funktionslogik und Zerfall einer terroristischen Organisation. Wiesbaden: Westdeutscher Verlag.

Thompson, Grahame/Jennifer Frances/Rosalind Levacic/Jeremy Mitchell (Hrsg.) (1991): Markets, Hierarchies and Networks. The Coordination of Social Life. London u.a..: Sage.

Ulfkotte, Udo (2003): Der Krieg in unseren Städten. Wie radikale Islamisten unsere Städte unterwandern. Frankfurt a.M.: Eichborn.

Verspagen, Bart/Claudia Werker (2003): The Invisible College of the Economics of Innovation and Technological Change. Manuskript. Eindhoven University of Technology.

Waldmann, Peter (1993): Terrorismus und Guerilla. Ein Vergleich organisierter antistaatlicher Gewalt in Europa und Lateinamerika. In: Jahrbuch Extremismus \& Demokratie 5, Bonn: Bouvier, S. 69-103.

Wege, Carl Anthony (1994): Hizbollah Organization. In: Studies in Conflict and Terrorism 17, S. 151-164.

Weick, Karl E./Karlene Roberts (1993): Collective Mind in Organizations: Heedful Interrelating on Flight Decks. In: Administrative Science Quarterly 38, S. 357-380.

Williamson, Oliver E. (1975): Markets and Hierarchies: Analysis and Antitrust Implications. A Study of the Economics of Internal Organization. New York: Free Press. 